\title{
Cerenkov-Activated Sticky Tag for In Vivo Fluorescence Imaging
}

\author{
Sudeep Das ${ }^{1}$, Katja Haedicke ${ }^{1}$, and Jan Grimm ${ }^{1-4}$ \\ ${ }^{1}$ Molecular Pharmacology Program, Memorial Sloan Kettering Cancer Center, New York, New York; ${ }^{2}$ Department of Radiology, \\ Memorial Sloan Kettering Cancer Center, New York, New York; ${ }^{3}$ Pharmacology Program, Weill Cornell Medical College, New York, \\ New York; and ${ }^{4}$ Department of Radiology, Weill Cornell Medical College, New York, New York
}

\begin{abstract}
A big challenge in the clinical use of Cerenkov luminescence (CL) imaging is its low signal intensity, which is several orders of magnitude below ambient light. Consequently, highly sensitive cameras, sufficient shielding from background light, and long acquisition times are required. To alleviate this problem, we hypothesized a strategy to convert the weak CL signal into a stronger fluorescence signal by using CL-activated formation of nitrenes from azides to locally fix a fluorescent probe in tissue by the formation of a covalent bond. CL-activated drug delivery was also evaluated using the same azide chemistry. The specific delivery of the CL-activated drug to cancer cells could reduce systemic toxicity, which is a limitation in chemotherapy. Methods: A cyanine-class near-infrared fluorescent dye, Cy7, and doxorubicin were synthetically attached to polyfluorinated aryl azide to form Cy7 azide and DOX azide, respectively. Fibrosarcoma cells were incubated with ${ }^{18} \mathrm{~F}-\mathrm{FDG}$ and exposed to Cy7 azide with subsequent fluorescence imaging. For CLactivated tagging in vivo, tumor-bearing mice were injected first with ${ }^{90} Y-D O T A-R G D$, targeting $\alpha_{v} \beta_{3}$ integrins, and then with the Cy7 azide. Fluorescence signal was imaged over time. Breast cancer cells were incubated with DOX azide and ${ }^{68} \mathrm{Ga}$, after which cell viability was quantified using an assay. Results: $\mathrm{CL}$ photoactivation of Cy7 azide in vitro showed significantly higher fluorescence signal from ${ }^{18} \mathrm{~F}-\mathrm{FDG}$-treated than untreated cells. In vivo, CL photoactivation could be shown by using the tumor-specific, integrin-targeting ${ }^{90} \mathrm{Y}$ DOTA-RGD and the localized activation of Cy7 azide. Here, localized $\mathrm{CL}$-induced fluorescence was detected in the tumors and remained significantly higher over several days than in tumors without CL. We also established as a next step CL-activated drug delivery of DOX azide by showing significantly decreasing cell viability of breast cancer cells in a CL dose-dependent manner in vitro using CL photoactivation of DOX azide. Conclusion: We were able to develop a CL-activated "sticky tag" that converts the low CL signal into a stable and long-lasting, highly intense fluorescence signal. This fluorescent footprint of the radioactive signal might be clinically used for intraoperative surgery. The CL-targeted drug delivery strategy may potentially be used for dual-step targeted therapy.
\end{abstract}

Key Words: Cerenkov luminescence; photoactivatable; azide; fluorescence; sticky tag; near-infrared optical imaging

J Nucl Med 2018; 59:58-65

DOI: 10.2967/jnumed.117.198549

Received Jul. 11, 2017; revision accepted Aug. 22, 2017.

For correspondence or reprints contact: Jan Grimm, Memorial Sloan Kettering Cancer Center, 1275 York Ave., Box 248, New York, NY 10065.

E-mail: grimmj@mskcc.org

Published online Sep. 14, 2017.

COPYRIGHT (C 2018 by the Society of Nuclear Medicine and Molecular Imaging.
O years as a robust optical modality in preclinical and clinical applications (1-6). When a charged subatomic particle passes through a medium at speeds higher than the speed of light in that medium, the particle polarizes nearby molecules. CL arises on subsequent relaxation of these molecules from the polarized state. Most isotopes that are already being used for nuclear imaging and radiotherapy generate CL (7). The CL from positron- or electronemitting radioisotopes can be used as an intrinsic source of light and suggests photoactivation of agents, overcoming the limited penetration and lack of accessibility of an external light source (8). However, the major limitation of CL is the low signal intensity and, depending on the isotope used, the ephemerality of the signal. The low signal intensity requires longer scanning times, which inherently could lower image quality because of subject movement during the imaging process. Also, for quickly decaying isotopes, the time restrictions for imaging are more constrained, especially if other imaging modalities, such as PET or SPECT, are being used concurrently, requiring time for their acquisition.

Radioisotopes are of course also used for targeted radiation therapy, which is often the first line of defense in the treatment of patients with thyroid cancer, metastatic prostate cancer, and lymphoma (9). Here, ${ }^{90} \mathrm{Y}$ is one of the preferred therapeutic isotopes because it is a pure, high-energy $\beta$-emitter (maximum energy, 2.3 MeV) without $\gamma$-radiation and enables a localized cell death (10). RGD-based small molecules labeled with ${ }^{90} \mathrm{Y}$ have been used for internal targeted radiotherapy, showing delayed growth of tumors $(11,12)$. In addition, a combination of targeted internal radiation therapy with chemotherapy has been shown to be superior in progression-free survival and overall survival to radiotherapy or chemotherapy alone in the treatment of high-grade gliomas and a wide variety of different cancers (13-17). However, the unspecific distribution of the chemotherapeutic drugs is the major reason for the toxicity of this combination $(18,19)$. To circumvent these side effects, this combination treatment modality needs to become more specific and selective to the tumor site.

To solve the abovementioned limitations in imaging and therapy, we hypothesized a mechanism by which CL could trigger the formation of a "sticky tag" to localize a CL-targeted fluorophore or a cytotoxic drug, as shown in Figure 1. The mechanism of formation of a sticky tag was adapted from polyfluorinated aryl azide chemistry, which has been shown to generate singlet nitrene species on irradiation with ultraviolet light (20). Photoaffinity ligands such as polyfluorinated aryl azides are preferable as they can be excited by the blue-weighted CL, leading to the generation 


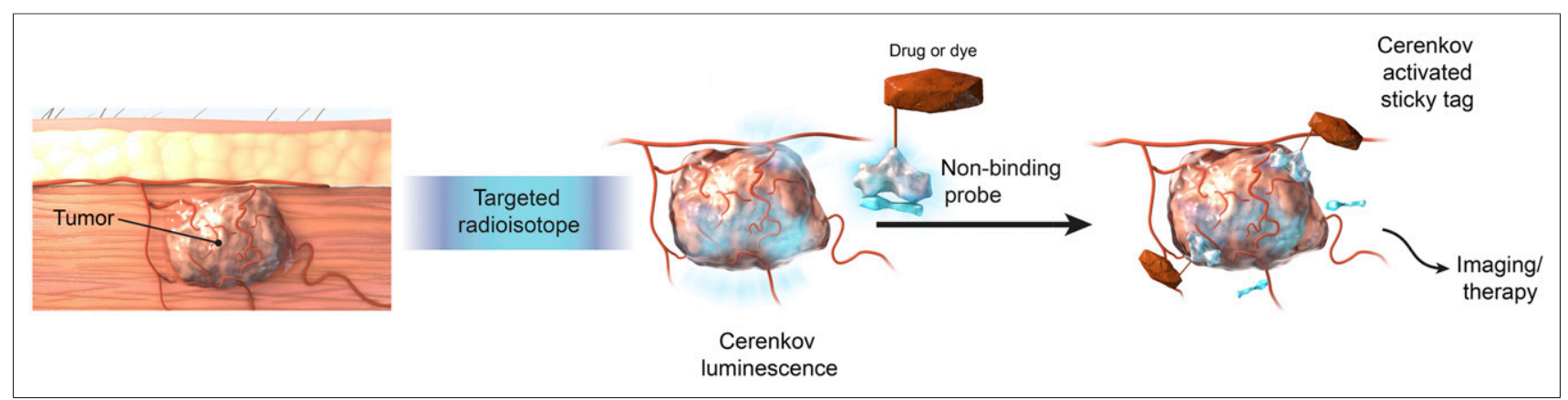

FIGURE 1. Principle of CL-activatable azide probes for targeting of tumor tissue.

of reactive singlet nitrene species, and the presence of fluorines on the aryl ring prevents intramolecular insertion (21). These nitrenes then immediately and irreversibly insert into $\mathrm{C}-\mathrm{H}$ and $\mathrm{N}-\mathrm{H}$ bonds of extracellular proteins or lipids by the formation of covalent bonds in an intermolecular fashion, acting like a sticky tag (22). Polyfluorinated aryl azide has been used previously for studying protein ligand binding interactions, identifying drug targets, and studying endogenous biologic molecules $(21,23)$. However, to our knowledge they have never been applied to an in vivo or CLactivated dye or drug delivery system as in our study.

To evaluate CL-activatable fluorescence imaging, polyfluorinated aryl azide is functionalized with a cyanine-class nearinfrared fluorescent dye, Cy7, to make Cy7 azide. CL-mediated localized activation and thus accumulation provide a durable fluorescence signal for standard fluorescence imaging. A Cy7 fluoride is used as a nonactivatable control dye in which the polyfluorinated aryl azides moiety of a Cy7 azide is synthetically replaced with fluorine. To induce the activation, ${ }^{18} \mathrm{~F}-\mathrm{FDG}$ or ${ }^{90} \mathrm{Y}-$ labeled DOTA-RGD peptide is used. Since ${ }^{90}$ Y-DOTA-RGD is targeted to $\alpha_{\mathrm{v}} \beta_{3}$-integrins expressed on the cancer cells as well as the neovasculature (24), the CL is generated at the tumor site, enabling a selective activation of the dye-labeled azide injected after free activity has cleared. Of course, by choosing other targeting moieties for the CL generator, one could target other structures (tumor cells, vascular plaques, inflammation). Here, a multitude of clinical radiotracers could be harvested for image-guided surgery but circumventing the shortcomings of CL by translating the CL signal with a single CL-activated fluorescent sticky tag into a fluorescent signal that allows for facile intraoperative imaging.

To evaluate a CL-activatable drug delivery system, a chemotherapeutic drug, doxorubicin, is functionalized with polyfluorinated aryl azide to make DOX azide. This strategy could essentially allow for a dual-step targeting strategy, separating the delivery of radioactivity from the delivery of a CL-activatable DOX azide and potentially reducing nonspecific cytotoxicity.

\section{MATERIALS AND METHODS}

\section{Reagents}

All chemicals, unless otherwise noted, were acquired from SigmaAldrich. The near-infrared dye sulfo-Cy7 $N$-hydroxysuccinimide (NHS) ester was purchased from Lumiprobe. Boc-1-amino-3,6-dioxa-8octanediamine was purchased from Chem-Impex International. DOTA-Glu-[cyclo(Arg-Gly-Asp-D-Phe-Lys) $]_{2}$ was purchased from Peptides International and is referred to as DOTA-RGD. Doxorubicin was purchased as an $\mathrm{HCl}$ salt from Selleckchem. ${ }^{18} \mathrm{~F}-\mathrm{FDG}$ was obtained as a saline solution from IBA Molecular through the Nuclear
Pharmacy at Memorial Sloan Kettering Cancer Center. ${ }^{90} \mathrm{YCl}_{3}$ was obtained as a $0.05 \mathrm{M} \mathrm{HCl}$ solution from Perkin Elmer. ${ }^{64} \mathrm{CuCl}_{2}$ was produced on the cyclotron at Washington University Medical School (model CS-15; Cyclotron Corp.) by the ${ }^{64} \mathrm{Ni}(p, n){ }^{64} \mathrm{Cu}$ reaction and purified to yield a specific activity of $7.4-14.8 \mathrm{GBq} / \mathrm{mg}$ in a $0.1 \mathrm{M}$ $\mathrm{HCl}$ solution. ${ }^{68} \mathrm{Ga}$ was eluted from a ${ }^{68} \mathrm{Ge} /{ }^{68} \mathrm{Ga}$ generator using $0.5 \mathrm{M} \mathrm{KOH}$, neutralized using $30 \% \mathrm{HCl}$, and mixed with phosphatebuffered saline (PBS) powder concentrate. (2,5-dioxopyrrolidin-1-yl)4azido-2,3,5,6-tetrafluorobenzoate (Fig. 2A, compound 1) was synthesized from 4-azido-2,3,5,6-tetrafluorobenzoic acid according to a previously published protocol (25). $\mathrm{N}$-(2-aminoethyl)-4-azido-2,3,5,6tetrafluorobenzamide (Supplemental Fig. 1, compound 2; supplemental materials are available at http://jnm.snmjournals.org) was synthesized from (2,5-dioxopyrrolidin-1-yl)4-azido-2,3,5,6-tetrafluorobenzoate according to a previously published protocol (26). All azide-containing syntheses were protected from exposure to direct light, as was the final product. High-performance liquid chromatography (HPLC) for qualitative analysis and quantitative purification was performed on a Shimadzu HPLC system equipped with LC20AD Prominence pumps, an SPD-M10AVP photodiode array detector, an FRC-10A fraction collector, and a DGU20A degasser. Reverse-phase HPLC was performed using an Atlantis T3 analytic column (100 $\mathrm{A}, 3 \mu \mathrm{m}, 4.6 \times 150 \mathrm{~mm}$; Waters) and an Atlantis T3 OBD semiprep column (100 $, 5 \mu \mathrm{m}, 19 \times 150 \mathrm{~mm}$; Waters), along with Milli-Q water ( $0.1 \%$ formic acid; MilliporeSigma) as solvent A and acetonitrile $(0.1 \%$ formic acid) as solvent $\mathrm{B}$.

Radiochemistry reactions were performed in Chelex (Bio-Rad) purified Milli-Q water. The radiolabeling was monitored using instant thin-layer chromatography (iTLC) by spotting on silicic acid-coated chromatography paper (Agilent Technologies), eluting with $50 \mathrm{mM}$ ethylenediaminetetraacetic acid ( $\mathrm{pH}$ 5.5), and analyzing the developed strip on a System 200 imaging scanner coupled to an Autochanger 1000 using Win-Scan radio-TLC software, version 2.2 (these last 3 all from Bioscan Inc.). Radiochemical purity was also determined using a Flow-RAM radio-HPLC detector (LabLogic Systems) with the HPLC described above, and signal was recorded using Shimadzu software. The radio-HPLC column and solvent setup was the same as that used in the nonradiosynthesis application. Activity was measured using a CRC-15R dose calibrator (Capintec).

\section{Cells and Animals}

Human fibrosarcoma cell line HT1080 and human breast cancer cell line MDA-MB-231 were obtained from the American Type Culture Collection. Human breast cancer cell line 4T1-luc2-GFP was obtained from Perkin Elmer. All cell lines were cultured using standard conditions.

All animal experiments were conducted in accordance with institutional guidelines and the protocol approved by the Institutional Animal Care and Use Committee. Female Hsd:athymic nude-Foxn $1^{n u}$ mice (7-9 wk old; weight, 18-20 g) were obtained from Envigo, and 


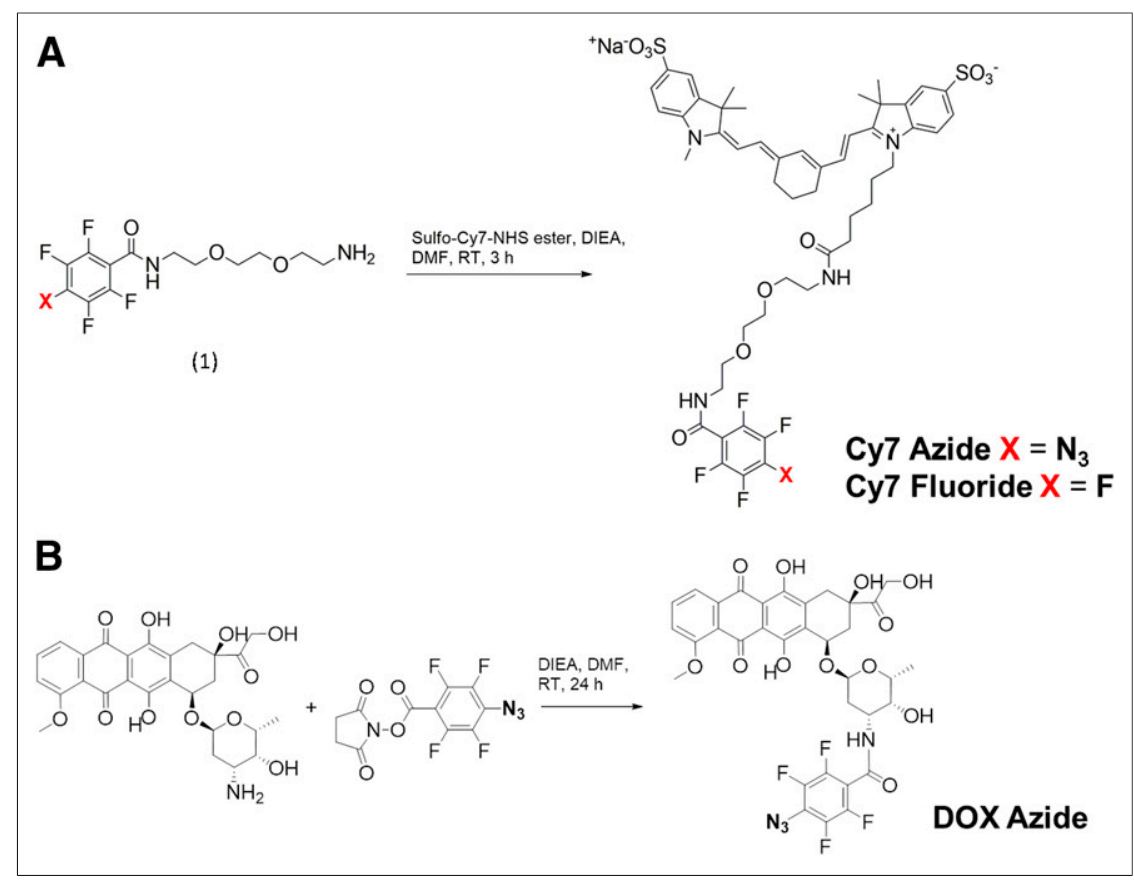

FIGURE 2. Synthesis of Cy7 azide and Cy7 fluoride (A) and of DOX azide (B). RT = room temperature. and added to an ice-cold solution of $2,3,4,5,6$ pentafluorobenzoyl chloride $(19.5 \mathrm{mg}, 0.085$ $\mathrm{mmol}$ ) in $0.1 \mathrm{~mL}$ of dichloromethane. Afterward, the reaction mixture was gradually warmed to room temperature and stirred for $2 \mathrm{~h}$. The reaction was then quenched with $0.1 \mathrm{~mL}$ of water and concentrated using rotary evaporation. Neat TFA was mixed with the crude product and stirred for $4 \mathrm{~h}$. The reaction mixture was concentrated using rotary evaporation and purified using HPLC to get free amine. Purified free amine (2.4 $\mathrm{mg}, 0.007 \mathrm{mmol}$ ) was dissolved in $21.4 \mu \mathrm{L}$ of dimethyl sulphoxide and mixed with sulfo-Cy7 NHS ester (5 mg, $0.006 \mathrm{mmol}$ ), DIEA (10.5 $\mu \mathrm{L}, 0.06 \mathrm{mmol}$ ), and DMF $(2 \mathrm{~mL})$. The reaction mixture was stirred for $3 \mathrm{~h}$, rotary evaporated, and purified using preparative HPLC, and the desired fractions were lyophilized.

\section{Synthesis of DOX Azide}

Doxorubicin $(5 \mathrm{mg}, 8.6 \mu \mathrm{M})$ was dissolved in $86.2 \mu \mathrm{L}$ of DMF and added to (2,5-dioxopyrrolidin-1-yl)4-azido-2,3,5,6-tetrafluorobenzoate $(10.3 \mathrm{mg}, 0.031 \mathrm{mmol})$ and DIEA (15 $\mu \mathrm{L}, 86 \mu \mathrm{M})$ and diluted by adding $250 \mu \mathrm{L}$ of DMF. The reaction mixture was all procedures were performed using 2\% isoflurane (Baxter Healthcare) as inhalation anesthesia. Xenografts of HT1080 cells were implanted by subcutaneous injection of $1.5 \times 10^{6}$ cells suspended in $100 \mu \mathrm{L}$ of Matrigel (BD Biosciences) in the left and right flanks of the mice. Experiments were performed when the tumors reached a volume of approximately $150 \mathrm{~mm}^{3}$. The mice were fed an irradiated nonfluorescent diet before fluorescence imaging to lower autofluorescence.

\section{Synthesis of Calcein Azide}

Calcein azide was synthesized by conjugating the free amine of the $N$-(2-aminoethyl)-4-azido-2,3,5,6-tetrafluorobenzamide (Supplemental Fig. 1, compound 2) to the activated carboxylic acid of calcein (21 mg, $0.034 \mathrm{mmol}$ ). Calcein was dissolved in $1 \mathrm{~mL}$ of dimethyl formamide (DMF) and cooled to $0^{\circ} \mathrm{C}$, followed by the addition of $N$-(3-dimethylaminopropyl)- $N N^{\prime}$-ethylcarbodiimide hydrochloride $(56 \mathrm{mg}$, $0.034 \mathrm{mmol})$ and NHS (9 mg, $0.08 \mathrm{mmol})$. After $30 \mathrm{~min}, \mathrm{~N}$-(2-aminoethyl)-4-azido-2,3,5,6-tetrafluorobenzamide (14 mg, $0.051 \mathrm{mmol})$ was added and stirred overnight at room temperature. The reaction mixture was diluted with water and washed with ethyl acetate, and the final product was isolated after lyophilization.

\section{Synthesis of Cy7 Azide}

Boc-1-amino-3,6-dioxa-8-octanediamine $(6.5 \mathrm{mg}, 0.026 \mathrm{mmol})$ and 2,5-dioxopyrrolidin-1-yl 4-azido-2,3,5,6-tetrafluorobenzoate $(10.3 \mathrm{mg}$, $0.031 \mathrm{mmol}$ ) were dissolved in $0.5 \mathrm{~mL}$ of DMF. $N, N$-diisopropylethylamine (DIEA) $(54 \mu \mathrm{L}, 0.31 \mathrm{mmol})$ was added and stirred for $1 \mathrm{~h}$ at room temperature. After the addition of $100 \mu \mathrm{L}$ of water, the reaction mixture was lyophilized. Neat TFA was added to the residue and stirred for $2 \mathrm{~h}$, after which the free amine was purified by preparative HPLC. Purified free amine $(2.48 \mathrm{mg}, 0.006 \mathrm{mmol})$ and sulfo-Cy7 NHS ester ( $5 \mathrm{mg}, 0.006 \mathrm{mmol})$ were dissolved in $2 \mathrm{~mL}$ of DMF. DIEA $(10.5 \mu \mathrm{L}$, $0.06 \mathrm{mmol}$ ) was added, and the reaction mixture was stirred for $2 \mathrm{~h}$, followed by purification using HPLC and lyophilization.

\section{Synthesis of Cy7 Fluoride (Nonactivatable Control)}

Boc-1-amino-3,6-dioxa-8-octanediamine (14 mg, $0.056 \mathrm{mmol})$ and DIEA $(22.2 \mu \mathrm{L}, 0.127 \mathrm{mmol})$ were dissolved in $0.2 \mathrm{~mL}$ of dichloromethane stirred for $24 \mathrm{~h}$ and purified using preparative HPLC, and the desired fractions were lyophilized.

\section{Synthesis of ${ }^{90} \mathrm{Y}-\mathrm{DOTA}-\mathrm{RGD}$}

A 200-MBq activity of ${ }^{90} \mathrm{YCl}_{3}$ in $9 \mu \mathrm{L}$ of $0.05 \mathrm{M} \mathrm{HCl}$ was added to a mixture of $27 \mu \mathrm{L}$ of sodium ascorbate $(20 \mathrm{mg} / \mathrm{mL}), 135 \mu \mathrm{L}$ of $200 \mathrm{mM}$ ammonium acetate ( $\mathrm{pH} 5.5)$, and $7.325 \mu \mathrm{L}$ of $1 \mathrm{mM}$ DOTA-RGD $(12.5 \mu \mathrm{g}, 7.3 \mathrm{nmol})$. The resulting reaction mixture was incubated in a ThermoMixer (Eppendorf) at $37^{\circ} \mathrm{C}$ with moderate mixing for $1 \mathrm{~h}$. Afterward, the radiochemical purity of ${ }^{90}$ Y-DOTA-RGD was verified using iTLC and radio-HPLC. A serum stability analysis was performed by adding $5 \mu \mathrm{L}(0.37 \mathrm{MBq})$ of ${ }^{90} \mathrm{Y}$-DOTA-RGD to $850 \mu \mathrm{L}$ of fetal bovine serum and determining the radiochemical purity at different time points using iTLC.

\section{Synthesis of ${ }^{64} \mathrm{Cu}-\mathrm{DOTA}-\mathrm{RGD}$}

A 53-MBq activity of ${ }^{64} \mathrm{CuCl}_{2}$ in $72 \mu \mathrm{L}$ of $0.1 \mathrm{M} \mathrm{HCl}$ was added to a mixture of $11.1 \mu \mathrm{L}$ of sodium ascorbate $(20 \mathrm{mg} / \mathrm{mL}), 55.5 \mu \mathrm{L}$ of $200 \mathrm{mM}$ ammonium acetate ( $\mathrm{pH} 5.5$ ), and $7.325 \mu \mathrm{L}$ of $1 \mathrm{mM}$ DOTARGD $(5.1 \mu \mathrm{g}, 3 \mathrm{nmol})$. The resulting reaction mixture was incubated in a ThermoMixer at $37^{\circ} \mathrm{C}$ with moderate mixing for $40 \mathrm{~min}$, after which the radiochemical purity of ${ }^{64} \mathrm{Cu}$-DOTA-RGD was verified using iTLC.

\section{Monitoring of Ultraviolet-Catalyzed Aryl Azide Photocleavage Using Absorption Spectra}

A $12-\mu \mathrm{g}$ quantity of 4-azido-2,3,5,6-tetrafluorobenzoic acid was dissolved in $1 \mathrm{~mL}$ of $\mathrm{CHCl}_{3}$ and irradiated using an ultraviolet-Cradiating germicidal Puritec HNS 30-watt G13 mercury lamp (Osram) at a distance of about $70 \mathrm{~cm}$. Ultraviolet-visible spectra were recorded after 2, 5, 10, 15, and 20 min of irradiation (Supplemental Fig. 2).

\section{In Vitro Activation of Calcein Azide Using Ultraviolet Light}

Four thousand MDA-MB-231 cells were seeded into each well of a 4-well chamber slide and incubated overnight to form a monolayer. On the following day, the cells were fixed with $4 \%$ paraformaldehyde for $15 \mathrm{~min}$ and washed once with PBS. Afterward, 2 wells were 
incubated with either PBS only, a $67 \mu \mathrm{g} / \mathrm{mL}$ solution of pure calcein, or a $67 \mu \mathrm{g} / \mathrm{mL}$ solution of calcein azide. Calcein- and calcein azidetreated cells were further divided into 2 subgroups. One well of each group was kept in the dark, and the other was ultraviolet-C-irradiated for 10 min using a germicidal Puritec HNS 30-watt G13 mercury lamp at a distance of about $70 \mathrm{~cm}$. Afterward, all slides were washed with PBS, and the cells were imaged using an Eclipse fluorescence microscope (exposure time $36 \mathrm{~ms}$; Nikon) to detect fluorescence of the activated calcein azide (Supplemental Fig. 3A). Quantitative analysis was performed using the signal-to-noise ratio calculated by NIS-Elements AR, version 4.0 (Nikon), for each condition $(n=3)$ (Supplemental Fig. 3B).

\section{In Vitro Activation of Cy7 Azide Using CL}

Trypsinized HT1080 cells $\left(2 \times 10^{6}\right)$ were washed with PBS and incubated with either $9.25 \mathrm{MBq}$ of ${ }^{18} \mathrm{~F}-\mathrm{FDG}$ or PBS $(n=3)$. After $30 \mathrm{~min}$, the cells were centrifuged at 1,000 rpm, the supernatant decanted, and the cells washed with PBS. A $10-\mu \mathrm{L}$ volume of $10 \mu \mathrm{M} \mathrm{Cy} 7$ azide was added and incubated for $2 \mathrm{~h}$. After incubation, the cells were washed with PBS and the fluorescence of the cells was measured using an IVIS Spectrum system (Perkin Elmer) with a 745-nm excitation filter and an 800-nm emission filter with binning of 4 , field of view of 24.4 , and $\mathrm{f} 2$ of $0.75 \mathrm{~s}$. Quantitative analysis was performed by drawing regions of interest over the cell pellets using Living Image software, version 4.2.

\section{Tumor Accumulation and Biodistribution of ${ }^{64} \mathrm{Cu}$-DOTA-RGD}

To investigate the tumor accumulation of ${ }^{64} \mathrm{Cu}$-DOTA-RGD over time, 3 HT1080 tumor-bearing mice were administered $11.1 \mathrm{MBq}$ of ${ }^{64} \mathrm{Cu}$-DOTA-RGD in sterile saline via retroorbital injection. Ten-minute static PET scans were acquired, followed by 2-min CT scans on an Inveon small-animal PET/CT scanner (Siemens) at 1, 3, 7, 10, 19, and $25 \mathrm{~h}$ after injection. Quantitative analysis of the tumor uptake was done by drawing a region of interest over the tumor using AsiPro VM software (Concorde Microsystems) and Inveon Research Workplace software (version 4.1; Siemens). For biodistribution analysis, 20 HT1080 tumor-bearing mice were administered $0.56 \mathrm{MBq}$ of ${ }^{64} \mathrm{Cu}-\mathrm{DOTA}-\mathrm{RGD}$ in sterile saline via retroorbital injection. Mice were sacrificed at $1,3,7,10,19$, and $25 \mathrm{~h}$ after injection ( 3 animals per time point) and dissected. Tumors, blood, heart, lung, liver, kidneys, stomach, intestine, muscle, and spleen were placed into tubes and weighed, and the activity was measured in a $\gamma$-counter (Wizard ${ }^{2}$; Perkin Elmer). The percentage of injected dose per gram of tissue $(\% \mathrm{ID} / \mathrm{g})$ was calculated for every organ $(n=3)$.

\section{In Vivo Activation of Cy7 Azide Using CL from 90Y-DOTA-RGD}

A 29.6-MBq activity of ${ }^{90} \mathrm{Y}$-DOTA-RGD in $100 \mu \mathrm{L}$ of PBS was administered to 5 tumor-bearing animals by retroorbital injection. Saline was injected into 4 more mice as a control. CL images were acquired using the IVIS Spectrum system from $5 \mathrm{~min}$ to $6 \mathrm{~h}$ after injection at different time points with an open emission filter, a blocked excitation filter, binning of 8 , and an $\mathrm{f} 1$ and exposure time of $30 \mathrm{~s}$. After $7 \mathrm{~h}, 100 \mu \mathrm{L}$ of $1 \mathrm{mM} \mathrm{Cy} 7$ azide were retroorbitally administered in the alternate eye of the previously injected 9 mice. In a separate control experiment, 5 mice were injected with $100 \mu \mathrm{L}$ of $1 \mathrm{mM}$ Cy7 fluoride at $7 \mathrm{~h}$ after a $29.6-\mathrm{MBq}$ injection of ${ }^{90}$ Y-DOTA$\mathrm{RGD}$, and 4 mice were injected with saline and $100 \mu \mathrm{L}$ of $1 \mathrm{mM}$ Cy 7 fluoride to determine nonspecific uptake of the control agent. Fluorescence images of all mice were acquired over time until $158 \mathrm{~h}$ after injection using the IVIS Spectrum system as described before. Quantitative analysis was done by drawing regions of interest over the tumors and the muscle, and the tumor-to-muscle ratio was calculated.
In Vitro Activation of DOX Azide Using CL

Five thousand 4T1 cells per well were seeded in a black 96-well plate with a clear bottom (Costar) and incubated overnight. On the next day, the medium was removed and the cells were covered with $50 \mu \mathrm{L}$ of fresh medium with or without $1 \mathrm{nmol}$ of DOX azide. Wells with and without the DOX azide were further treated with or without 3.7 MBq and $11.1 \mathrm{MBq}$ of ${ }^{68} \mathrm{Ga}$ in PBS buffer (3 wells per condition). The cells were incubated for $48 \mathrm{~h}$. Afterward, a viability assay was performed by adding $10 \mu \mathrm{L}$ of AlamarBlue reagent (Invitrogen) and $90 \mu \mathrm{L}$ of medium into each well and incubating for $4 \mathrm{~h}$. The fluorescence of the plate was recorded using a plate reader (SpectraMax M5; Molecular Devices) with an excitation at $570 \mathrm{~nm}$ and emission at $585 \mathrm{~nm}$ and analyzed quantitatively.

\section{Statistical Analysis}

Data and statistical analyses were performed using Prism (version 5.01; GraphPad Software, Inc.). Data were analyzed using the unpaired, 2-tailed Student $t$ test. Differences at the 95\% confidence level $(P<0.05)$ were considered to be statistically significant. For time-series data, statistical significance was evaluated for each time slice, and a significant difference over the entire time series was represented using square brackets at the tail of the plot.

\section{RESULTS}

\section{Synthesis and Characterization of Cy7 Azide, Cy7 Fluoride, and DOX Azide}

The Cy7 azide was synthesized by coupling sulfo-Cy7 NHS ester to the free amine (Fig. 2A, compound 1, $\mathrm{X}=\mathrm{N}_{3}$ ), and the desired product was obtained after preparative HPLC with greater than $90 \%$ yield. LCMS showed that the product was more than $98 \%$ pure, with a retention time of $5.38 \mathrm{~min}$. Mass spectroscopy showed a peak at $1,056.40 \mathrm{~m} / \mathrm{z}[\mathrm{M}+\mathrm{H}]^{+}$. The Cy7 fluoride was synthesized by coupling the Cy7 NHS ester to the free amine (Fig. $2 \mathrm{~A}$, compound $1, \mathrm{X}=\mathrm{F}$ ), and the desired product was obtained after preparative HPLC with greater than $90 \%$ yield. LCMS showed that the product was more than $98 \%$ pure, with a retention time of $5.45 \mathrm{~min}$. Mass spectroscopy showed a peak at 1,033.30 $\mathrm{m} / \mathrm{z}[\mathrm{M}+\mathrm{H}]^{+}$. DOX azide was prepared by coupling free amine of doxorubicin with 4-azido-2,3,5,6-tetrafluorobenzoyl chloride in greater than $99 \%$ yield, as shown in Figure 2B. LCMS revealed that the product was more than $98 \%$ pure, with a retention time of $7.25 \mathrm{~min}$. Mass spectroscopy showed a peak at $783.0 \mathrm{~m} / \mathrm{z}$ $[\mathrm{M}+\mathrm{Na}]^{+}$.

\section{Synthesis of ${ }^{90}$ Y-DOTA-RGD and ${ }^{64} \mathrm{Cu}$-DOTA-RGD}

The radiolabeling of DOTA-RGD with ${ }^{90} \mathrm{YCl}_{3}$ was successful, and a high specific activity of $27.4 \mathrm{MBq} / \mathrm{nmol}$ was achieved. ${ }^{90} \mathrm{Y}-$ DOTA-RGD was prepared with a radiochemical purity of more than $99 \%$, as shown by the $\mathrm{R}_{\mathrm{f}} 0$ peak on iTLC (Supplemental Fig. 4A) and a retention time of $7.7 \mathrm{~min}$ on radio-HPLC (Supplemental Fig. 4B). As a control, free ${ }^{90} \mathrm{Y}$ was shown to have an $\mathrm{R}_{\mathrm{f}}$ of 0.86 on iTLC (Supplemental Fig. 4C). The serum stability study with ${ }^{90}$ Y-DOTA-RGD showed that the radiochemical purity was retained over $6 \mathrm{~d}$ (Supplemental Fig. 4D).

\section{Monitoring Photoactivation of 4-Azido-2,3,5,6- Tetrafluorobenzoic Acid}

Cumulative ultraviolet-C doses to 4-azido-2,3,5,6-tetrafluorobenzoic acid showed a gradual decrease in the absorbance at $265 \mathrm{~nm}$ and more tailing above $310 \mathrm{~nm}$ (Supplemental Fig. 2). The isosbestic point for the photochemical transformation was found to be $290 \mathrm{~nm}$. 


\section{In Vitro Photoactivation of Calcein Azide}

Incubation of MDA-MB-231 cells with calcein azide led to a detectable fluorescence in those cells additionally treated with ultraviolet light (Supplemental Fig. 3A). In comparison, cells without ultraviolet treatment or the pure calcein control dye did not show a fluorescence signal. After quantification, the fluorescence signal-to-noise ratio of the cells treated with calcein azide and ultraviolet light was 3.5 times higher $(P<0.0001)$ than that of the cells treated with calcein azide only, at $15.56 \pm 0.03$ and $3.55 \pm 0.06$, respectively (Supplemental Fig. 3B). There was no autofluorescence of the untreated cells themselves, of the cells treated with pure calcein with or without ultraviolet light, or of the cells treated with calcein azide only.

In Vitro Activation of Cy7 Azide Using CL

Two million HT1080 cells incubated with ${ }^{18} \mathrm{~F}-\mathrm{FDG}$ had an average uptake of $4.33 \pm 0.07 \mathrm{MBq}$ of ${ }^{18} \mathrm{~F}-\mathrm{FDG}$ per cell pellet (2.22 Bq/cell). Subsequent incubation with Cy7 azide resulted in a strong fluorescence from the ${ }^{18} \mathrm{~F}-\mathrm{FDG}$-incorporated cell pellets, compared with the pellets incubated with $\mathrm{Cy} 7$ azide only (Fig. $3 \mathrm{~A})$. The quantitative analysis confirmed the observation by revealing a significantly higher fluorescence signal for the cells incubated with ${ }^{18}$ F-FDG and $\mathrm{Cy} 7$ azide than for the cells incubated with $\mathrm{Cy} 7$ azide alone, with average radiant efficiencies of $(7.770 \pm 0.348) \times 10^{7}$ and $(3.993 \pm 0.071) \times 10^{7}$, respectively $(P=0.0004)$ (Fig. 3B).

\section{Tumor Accumulation and Biodistribution of ${ }^{64}$ Cu-DOTA-RGD}

The PET/CT images at different time points after intravenous injection of ${ }^{64} \mathrm{Cu}$-DOTA-RGD showed a rapid accumulation of ${ }^{64} \mathrm{Cu}$-DOTA-RGD at the tumor site, as was also revealed by the quantitative analysis of these images (Fig. 4A). Furthermore, the biodistribution studies quantified the $\% \mathrm{ID} / \mathrm{g}$ for each tumor to be $8.1 \pm 1.8$ at $1 \mathrm{~h}$ after injection, $12.5 \pm 4.1$ at $3 \mathrm{~h}, 9.3 \pm 1.5$ at $7 \mathrm{~h}$,

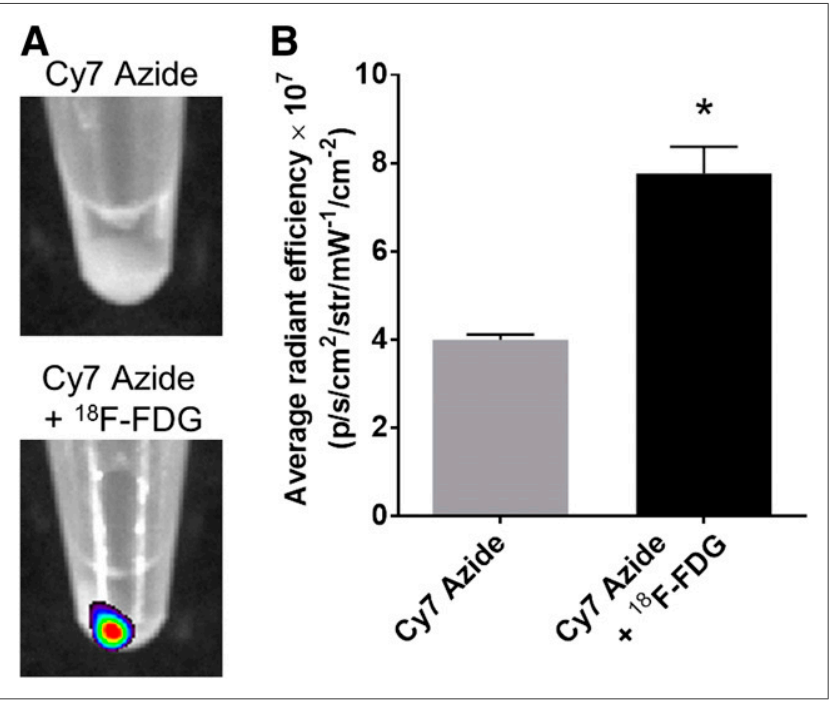

FIGURE 3. CL-activated cell labeling using Cy7 azide. (A) Fluorescence images of HT1080 cell pellets incubated with ${ }^{18} \mathrm{~F}-\mathrm{FDG}$ and Cy7 azide or Cy7 azide alone. (B) Quantitative analysis of $\mathrm{HT} 1080$ cell pellet fluorescence signal after incubation with Cy7 azide alone or together with ${ }^{18} \mathrm{~F}-\mathrm{FDG}(P=0.0004)$. IVIS Spectrum system was used for fluorescence imaging (emission filter, $800 \mathrm{~nm}$; excitation filter, $745 \mathrm{~nm}$; epiillumination; bins, 4; field of view, 24.4; f2, $0.75 \mathrm{~s}$ ). ${ }^{*} P<0.05$.

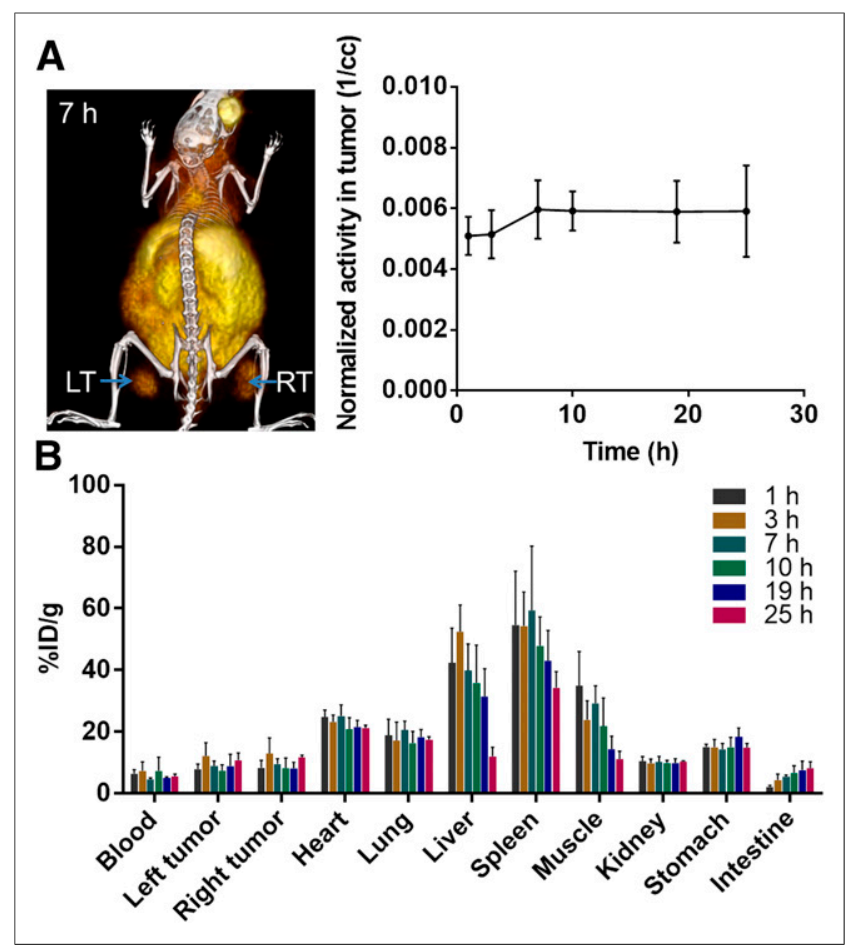

FIGURE 4. In vivo PET/CT imaging and biodistribution studies of ${ }^{64} \mathrm{Cu}$ DOTA-RGD. (A) Representative PET/CT image with left tumor (LT) and right tumor (RT) at $7 \mathrm{~h}$ after injection of ${ }^{64} \mathrm{Cu}-\mathrm{DOTA}-\mathrm{RGD}$. Graph shows quantitative analysis of activity in tumor as function of time. (B) Biodistribution of ${ }^{64} \mathrm{Cu}$-DOTA-RGD at different time points after injection as $\% \mathrm{ID} / \mathrm{g}$ of organ.

$7.8 \pm 2.2$ at $10 \mathrm{~h}, 8.5 \pm 2.7$ at $19 \mathrm{~h}$, and $11.3 \pm 1.5$ at $25 \mathrm{~h}, \mathrm{a}$ pattern that shows a rapid and steady uptake in the tumors (Fig. 4B). The conjugate was excreted mostly through the liver, with $42.4 \pm$ $9.7 \% \mathrm{ID} / \mathrm{g}$ at $1 \mathrm{~h}$ dropping to $12.0 \pm 2.4 \% \mathrm{ID} / \mathrm{g}$ at $25 \mathrm{~h}$.

\section{Imaging of ${ }^{90}$ Y-DOTA-RGD and In Vivo CL-Activated Accumulation of Cy7 Azide}

Mice injected with ${ }^{90}$ Y-DOTA-RGD showed a rapid and specific accumulation of activity in the tumor at $6 \mathrm{~h}$ after injection (Fig. 5A). The corresponding quantitative analysis showed an increase in the tumor CL signal within the first $2 \mathrm{~h}$ after injection (Fig. 5B). The CL signal stayed at this level up to $6 \mathrm{~h}$ after injection. Control mice received just saline injection before the Cy7 azide injection and thus lacked the CL-emitting radioisotope required to activate the azide. Mice that were injected with the $\mathrm{Cy} 7$ azide and ${ }^{90}$ Y-DOTA-RGD demonstrated a significantly higher fluorescence within the tumors (site of CL generation) than did the control mice (average radiant efficiency at $36 \mathrm{~h}$, $5.0 \times 10^{9} \pm 1.1 \times 10^{9}$ vs. $\left.2.4 \times 10^{9} \pm 0.4 \times 10^{9} ; P<0.0002\right)$ (Figs. 5C and 5D). Even $6.5 \mathrm{~d}(153 \mathrm{~h})$ after $\mathrm{Cy} 7$ azide injection, fluorescence within the tumors remained significantly higher and more stable than in the tumors of control mice $\left(1.6 \times 10^{9} \pm 0.5 \times\right.$ $10^{9}$ vs. $\left.0.8 \times 10^{9} \pm 0.2 \times 10^{9} ; P<0.002\right)$. Plotting the tumorto-muscle ratios of the Cy7 fluorescence for ${ }^{90}$ Y-DOTA-RGDtreated and -untreated mice also helped visualize the contrast enhancement within the tumor and showed maximum contrast to be at $72 \mathrm{~h}$ (Fig. 5E).

As a second control, we used a nonactivatable Cy7 fluoride control dye. Animals injected with ${ }^{90}$ Y-DOTA-RGD and Cy7 


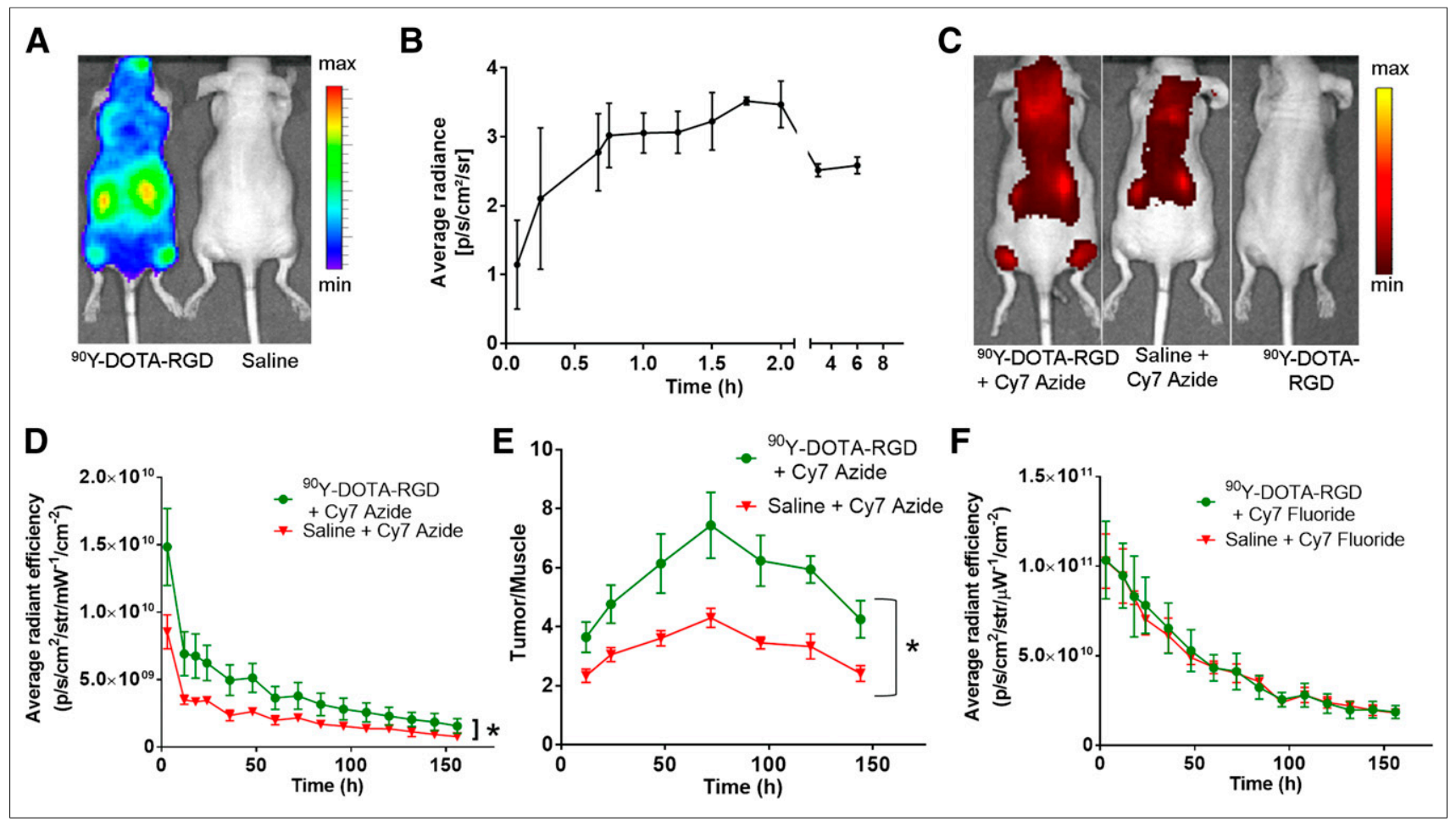

FIGURE 5. CL-activated tumor labeling using Cy7 azide. (A) CL images of mice injected with ${ }^{0} \mathrm{Y}-\mathrm{DOTA}-\mathrm{RGD}$ (6-h time point). (B) Quantitative analysis of $\mathrm{CL}$ radiance of tumors $6 \mathrm{~h}$ after injection of ${ }^{90} \mathrm{Y}$-DOTA-RGD. (C) Fluorescence images of mice injected with Cy7 azide $7 \mathrm{~h}$ after ${ }^{90} \mathrm{Y}-\mathrm{DOTA}-\mathrm{RGD}$ or saline injection, or ${ }^{90}$ Y-DOTA-RGD alone. (D) Quantitative analysis of fluorescence of tumors with Cy7 azide with and without ${ }^{90} \mathrm{Y}-\mathrm{DOTA}-\mathrm{RGD}$ over time $(P<$ 0.05). (E) Quantitative plot of tumor-to-muscle ratio in mice with ${ }^{90}$ Y-DOTA-RGD-activated Cy7 azide compared with saline control as function of time $(P<$ 0.05). (F) Quantitative analysis of fluorescence of tumors from mice injected with Cy7 fluoride control $7 \mathrm{~h}$ after ${ }^{90}$ Y-DOTA-RGD or saline injection over time. IVIS Spectrum system was used for optical imaging (CLI: emission filter, open; excitation filter, blocked; bins [medium; HR], 8; field of view, 24.4; f1, $30 \mathrm{~s}$ ) (fluorescence imaging: emission filter, $800 \mathrm{~nm}$; excitation filter, $745 \mathrm{~nm}$; epillumination; bins [high resolution; HR], 4; field of view, 24.4; f2, $0.75 \mathrm{~s}$ ).

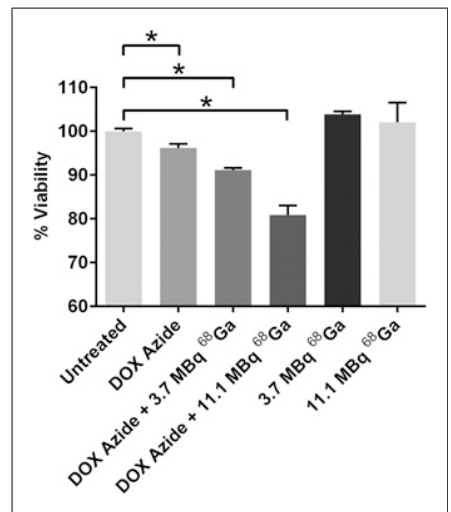

FIGURE 6. Dose-dependent CLactivated in vitro therapy using DOX azide. Viability is shown for $4 \mathrm{~T} 1$ cells treated with $1 \mathrm{nmol}$ of DOX azide and different doses of ${ }^{68} \mathrm{Ga}$. ${ }^{*} P<0.05$. only $3.8 \%$, compared with untreated cells $(P=0.0034)$ (Fig. 6). In comparison, treatment of the cells with DOX azide in combination with 3.7 or $11.1 \mathrm{MBq}$ of ${ }^{68} \mathrm{Ga}$ decreased the cell viability significantly by $9 \%(P<0.0001)$ and $20 \%(P=0.0001)$, respectively, compared with the untreated control. A treatment with 3.7 or $11.1 \mathrm{MBq}$ of ${ }^{68} \mathrm{Ga}$ alone did not affect the viability of the cells. This pilot demonstration suggests the possible further application of our approach. fluoride had no significant fluorescence enhancement in their tumors, in comparison to mice injected with Cy7 fluoride alone (average radiance fluorescence, $6.6 \times 10^{10} \pm$ $1.4 \times 10^{10}$ vs. $6.1 \times 10^{10} \pm$ $0.982 \times 10^{10}$, respectively, at 36 h) (Fig. 5F).

\section{In Vitro Activation of DOX Azide Using CL}

To demonstrate the possibility of developing a therapy based on this application, we developed an activatable doxorubicin analog. Treatment of MDA-MB-231 cells with DOX azide alone reduced the cell viability by

,

\section{DISCUSSION}

Our study demonstrated that attaching an azide group to various molecules leads to a specific and localized targeted delivery of those molecules to CL-emitting tumors. This strategy was successful with both CL-activatable azide conjugated to fluorescent dyes and chemotherapeutics. With this method, we converted a low CL signal into a stronger, long-lasting fluorescence signal that will facilitate the use of radiotracers for intraoperative imaging. We also showed enhanced cytotoxicity after the combination of CL with an activatable drug.

In our first experiments, we showed that the azide could be cleaved using ultraviolet light and monitored using ultraviolet-visible spectra. Also using ultraviolet light, we showed in vitro activation of calcein azide. We showed an enhanced delivery of calcein using ultraviolet light to activate calcein azide on tumor cells. Hence, in the presence of ultraviolet light, our calcein azide was also found to be bound to the tumor cells. Pure calcein was used as a control to show that the dye alone did not result in any accumulation with or without ultraviolet light. This finding points out that the azide moiety is essential for the attachment to the cells.

After showing proof of concept using ultraviolet light, we used CL to activate Cy7 azide. The accumulation of ${ }^{18}$ F-FDG in HT1080 cells could be seen by virtue of the Warburg effect (27). On incubation of the ${ }^{18} \mathrm{~F}-\mathrm{FDG}$-enriched cells with $\mathrm{Cy} 7$ azide, the $\mathrm{CL}$ activated the azide moiety, generating nitrenes and resulting in binding of the Cy7 construct to the cell surface. Cells without ${ }^{18}$ F-FDG had a significantly lower Cy7 signal, confirming our proposed mechanism. 
In vivo experiments were performed with ${ }^{90} \mathrm{Y}$ as the source of $\mathrm{CL}$, as this isotope produces more CL than other isotopes because of the emission of energetic electrons (28). The attachment of DOTA-RGD made this CL source highly specific through targeting of $\alpha_{V} \beta_{3}$ integrins on the tumor (29). The tumor-targeting efficiency of the peptide was quantified by biodistribution studies using ${ }^{64} \mathrm{Cu}$-labeled DOTA-RGD. Instead of the ${ }^{90} \mathrm{Y}$ that was used for $\mathrm{CL}$ activation of the azide, we switched to ${ }^{64} \mathrm{Cu}$ because of its usability in PET imaging and $\gamma$-counting. This quantification lets us gauge the tumor uptake of ${ }^{90} \mathrm{Y}-\mathrm{DOTA}-\mathrm{RGD}$ for CL activation of Cy7 azide activation. After accumulation of ${ }^{90}$ Y-DOTA-RGD at the tumor site, an enhanced labeling with $\mathrm{Cy} 7$ azide over time was detected in only these tumors, not in control tumors, which did not receive ${ }^{90}$ Y-DOTA-RGD. The retained fluorescence contrast even after several days indicates a stable covalent binding of the dye to the CL-enhanced tissue. The use of the inactive Cy7 fluoride instead of the active azide did not lead to a CL-dependent accumulation of fluorescence within the tumor, demonstrating that the presence of the azide is necessary for the accumulation of the dye. This indeed clarifies that the phenomenon we observed is truly a CL-triggered activation.

In future experiments, the fluorescence output gained from the Cy7 azide in the CL-enhanced tissue could be used for fluorescence-guided intraoperative resection. The near-infrared fluorescence probes are particularly important in clinical application because of their greater penetration depth and low autofluorescence. Translating CL into long-lasting fluorescence circumvents the disadvantage of the low signal intensity of the CL and the need for radioactivity. Surgeons could inject first the radiotracer and then the fluorescent CL-activated sticky tag. Once the activity has decreased or fully decayed, the surgeon can perform surgery, using the fluorescent tag for image-guided surgery. This technique can be used with the multitude of existing radiotracers, requiring only one azide label (one-stop shop), thus preserving the advantage of having many clinical tracers to mark tumors. The detection of cancer using such targeted fluorophores could possibly improve survival rates and outcomes in patients (30).

Using this system, we also investigated its possible application for a therapeutic approach. We introduced a CL-triggered, targeted drug delivery system by demonstrating a significant CL dosedependent increase in toxicity of DOX azide to breast cancer cells. This finding can be particularly useful in therapy settings that combine radiotherapy (as additional CL source) and chemotherapy. A multistep drug delivery strategy has previously been shown to be better for patients receiving combination therapy rather than chemotherapy or radiotherapy alone (17). Because DOX azide alone had a minor toxicity effect on the cells, the compound needs to be improved. However, a significantly higher toxicity was attained in combination with CL. Thus, the delivered drug is shown to benefit from the advantage that the azide version of the drug effectively is a pro-drug, activated only in the presence of CL excitation. This characteristic could increase the therapeutic efficacy because of the CL-activated targeting. The fact that the used ${ }^{68} \mathrm{Ga}$ doses showed no toxicity is promising for the potential use of even higher doses and the generation of more CL. The use of ${ }^{68} \mathrm{Ga}$ is not directly translatable but could easily be conjugated to DOTA-RGD, similar to the ${ }^{90} \mathrm{Y}$ conjugation techniques that we have shown and used to create targeted agents. The modest drop in cell viability calls for further research into more effective photochemistry and drugazide conjugates. Nonetheless, the indications for this new modality of small-molecule drug delivery are very promising.

\section{CONCLUSION}

We were able to photoactivate probes for tumor imaging and therapy by using CL. We could specifically activate Cy7 dyelabeled azides in vitro and in vivo to convert a low-intensity CL signal into a higher and long-lasting fluorescence signal. This conversion is suitable for circumventing the known disadvantages of CL imaging. Furthermore, we could introduce the first CLphotoactivated drug delivery system. Our targeting strategy can be used to enable a more specific fluorescent intraoperative resection or to assess tissue radiation dose. With our approach, we could successfully establish a new CL application in the field of tumor imaging and therapy.

\section{DISCLOSURE}

This work was supported by the National Institute of Health (R01EB014944 and R01 CA183953 to Jan Grimm and the MSKCC NIH Core Grant P30-CA008748). No other potential conflict of interest relevant to this article was reported.

\section{ACKNOWLEDGMENTS}

We thank Drs. Jason Lewis, Nagavarakishore Pillarsetty, and Thomas Reiner for help with radiochemistry. Also, we thank the MSKCC Radiopharmacy, Small-Animal Imaging Core Facility, Nuclear Magnetic Resonance Analytical Core Facility, and Design and Creative Services.

\section{REFERENCES}

1. Robertson R, Germanos MS, Li C, Mitchell GS, Cherry SR, Silva MD. Optical imaging of Cerenkov light generation from positron-emitting radiotracers. Phys Med Biol. 2009;54:N355-N365.

2. Thorek DL, Robertson R, Bacchus WA, et al. Cerenkov imaging: a new modality for molecular imaging. Am J Nucl Med Mol Imaging. 2012;2:163-173.

3. Thorek DLJ, Riedl CC, Grimm J. Clinical Cerenkov luminescence imaging of ${ }^{18}$ F-FDG. J Nucl Med. 2014;55:95-98.

4. Thorek DLJ, Ogirala A, Beattie BJ, Grimm J. Quantitative imaging of disease signatures through radioactive decay signal conversion. Nat Med. 2013;19:13451350 .

5. Thorek DL, Das S, Grimm J. Molecular imaging using nanoparticle quenchers of Cerenkov luminescence. Small. 2014;10:3729-3734.

6. Czupryna J, Kachur AV, Blankemeyer E, et al. Cerenkov-specific contrast agents for detection of $\mathrm{pH}$ in vivo. $\mathrm{J} \mathrm{Nucl} \mathrm{Med.} \mathrm{2015;56:483-488.}$

7. Das S, Thorek DL, Grimm J. Cerenkov imaging. Adv Cancer Res. 2014;124: 213-234.

8. Ran C, Zhang Z, Hooker J, Moore A. In vivo photoactivation without "light": use of Cherenkov radiation to overcome the penetration limit of light. Mol Imaging Biol. 2012;14:156-162.

9. Zukotynski K, Jadvar H, Capala J, Fahey F. Targeted radionuclide therapy: practical applications and future prospects. Biomark Cancer. 2016;8:35-38.

10. Chinol M, Paganelli G, Sudati F, Meares C, Fazio F. Biodistribution in tumourbearing mice of two ${ }^{90} \mathrm{Y}$-labelled biotins using three-step tumour targeting. Nucl Med Commun. 1997;18:176-182.

11. Hnatowich DJ, Virzi F, Doherty PW. DTPA-coupled antibodies labeled with yttrium-90. J Nucl Med. 1985;26:503-509.

12. Janssen ML, Oyen WJ, Dijkgraaf I, et al. Tumor targeting with radiolabeled $\alpha v \beta 3$ integrin binding peptides in a nude mouse model. Cancer Res. 2002;62:6146-6151.

13. Sofou S. Radionuclide carriers for targeting of cancer. Int J Nanomedicine. 2008;3:181-199.

14. Kong G, Johnston V, Ramdave S, Lau E, Rischin D, Hicks RJ. High-administered activity In-111 octreotide therapy with concomitant radiosensitizing 5FU chemotherapy for treatment of neuroendocrine tumors: preliminary experience. Cancer Biother Radiopharm. 2009;24:527-533.

15. DeNardo SJ. Combined molecular targeting for cancer therapy: a new paradigm in need of molecular imaging. J Nucl Med. 2006;47:4-5. 
16. Rusckowski M, Wang Y, Blankenberg FG, Levashova Z, Backer MV, Backer JM. Targeted $\mathrm{scVEGF} /{ }^{177} \mathrm{Lu}$ radiopharmaceutical inhibits growth of metastases and can be effectively combined with chemotherapy. EJNMMI Res. 2016;6:4.

17. Lawrence TS, Haffty BG, Harris JR. Milestones in the use of combined-modality radiation therapy and chemotherapy. J Clin Oncol. 2014;32:1173-1179.

18. Al-Sarraf M, LeBlanc M, Giri PG, et al. Chemoradiotherapy versus radiotherapy in patients with advanced nasopharyngeal cancer: phase III randomized intergroup study 0099. J Clin Oncol. 1998;16:1310-1317.

19. Penack O, Becker C, Buchheidt D, et al. Management of sepsis in neutropenic patients: 2014 updated guidelines from the Infectious Diseases Working Party of the German Society of Hematology and Medical Oncology (AGIHO). Ann Hematol. 2014;93:1083-1095.

20. Gritsan NP, Gudmundsdóttir AD, Tigelaar D, et al. A laser flash photolysis and quantum chemical study of the fluorinated derivatives of singlet phenylnitrene. J Am Chem Soc. 2001;123:1951-1962.

21. Lapinsky DJ. Tandem photoaffinity labeling-bioorthogonal conjugation in medicinal chemistry. Bioorg Med Chem. 2012;20:6237-6247.

22. Schnapp KA, Poe R, Leyva E, Soundararajan N, Platz MS. Exploratory photochemistry of fluorinated aryl azides: implications for the design of photoaffinity labeling reagents. Bioconjug Chem. 1993;4:172-177.
23. Xia Y, Peng L. Photoactivatable lipid probes for studying biomembranes by photoaffinity labeling. Chem Rev. 2013;113:7880-7929.

24. Ruoslahti E, Pierschbacher MD. New perspectives in cell adhesion: RGD and integrins. Science. 1987;238:491-497.

25. Marchand-Brynaert J, Welle A, Billard F. Tri- and tetravalent photoactivable cross-linking agents. Synthesis. 2012;44:2249-2254.

26. Rajagopalan R, Kuntz RR, Sharma U, Volkert WA, Pandurangi RS. Chemistry of bifunctional photoprobes. 6. Synthesis and characterization of high specific activity metalated photochemical probes: development of novel rhenium photoconjugates of human serum albumin and Fab fragments. J Org Chem. 2002;67: 6748-6757.

27. Warburg O. On the origin of cancer cells. Science. 1956;123:309-314.

28. Carpenter CM, Ma X, Liu H, et al. Cerenkov luminescence endoscopy: improved molecular sensitivity with beta-emitting radiotracers. J Nucl Med. 2014;55: 1905-1909.

29. Chen X, Conti PS, Moats RA. In vivo near-infrared fluorescence imaging of integrin $\alpha_{v} \beta_{3}$ in brain tumor xenografts. Cancer Res. 2004;64:8009-8014.

30. Zhang RR, Schroeder AB, Grudzinski JJ, et al. Beyond the margins: real-time detection of cancer using targeted fluorophores. Nat Rev Clin Oncol. 2017;14: 347-364. 